\title{
Effects of Guhanyangshengjing Tablet on Testosterone Synthesis and Expression of SYCP3 in the Testis of Aging Male Rats
}

\author{
Zhijun Zang ${ }^{*}$, Yong $\mathrm{Gao}^{2}$, Suyun $\mathrm{Ji}^{3}$, Meihua Jiang ${ }^{4}$ \\ ${ }^{1}$ Department of Infertility and Sexual Medicine, The Third Affiliated Hospital, Sun Yat-sen University, \\ Guangzhou, China \\ ${ }^{2}$ Reproductive Medicine Centre and Guangdong Provincial Key Laboratory of Reproductive Medicine, \\ The First Affiliated Hospital, Sun Yat-sen University, Guangzhou, China \\ ${ }^{3}$ Department of Dermatology, Guangdong Provincial Dermatology Hospital, Guangzhou, China \\ ${ }^{4}$ Department of Anatomy, Zhongshan School of Medicine, Sun Yat-sen University, Guangzhou, China \\ Email: *zangzhijun@163.com
}

Received 6 May 2016; accepted 29 May 2016; published 2 June 2016

Copyright (C) 2016 by authors and Scientific Research Publishing Inc.

This work is licensed under the Creative Commons Attribution International License (CC BY).

http://creativecommons.org/licenses/by/4.0/

(c) (7) Open Access

\begin{abstract}
Objective: The objective of this article is to investigate the effect of Guhanyangshengjing Tablet (GT) on expression of synaptonemal complex protein 3 (SYCP3), a meiotic marker, in the testis tissue of aging male rats. Methods: Forty aging male rats were randomly assigned into 4 equal groups ( $\mathrm{n}=10$ per group). Rats in each group were treated with GT at dose of 0 (control), $1.5 \mathrm{~g} / \mathrm{kg}$, $3.0 \mathrm{~g} / \mathrm{kg}$ or $4.5 \mathrm{~g} / \mathrm{kg}$ respectively by gavage daily for 30 days. At the end of the experiment, all animals were sacrificed and the blood samples were drawn to evaluate serum testosterone levels. The reproductive organs of each rat were taken and weighted. The right testis of each rat was removed for the analysis of intratesticular testosterone (ITT) concentrations, and the left one was used for immunohistochemical staining. Results: Compared with the control, reproductive organs' weights, serum testosterone levels, ITT concentrations, quality of sperm, and expression of SYCP3 in the GT-treated groups were all improved in a dose-dependent manner. Conclusion: GT can improve testosterone synthesis and promote spermatogenesis simultaneously, indicating that GT is suitable for late-onset hypogonadism $(\mathrm{LOH})$ patients with fertility requirements.
\end{abstract}

\section{Keywords}

Guhanyangshengjing Tablet (GT), Synaptonemal Complex Protein 3, Testosterone Synthesis,

"Corresponding author.

How to cite this paper: Zang, Z.J., Gao, Y., Ji, S.Y. and Jiang, M.H. (2016) Effects of Guhanyangshengjing Tablet on Testosterone Synthesis and Expression of SYCP3 in the Testis of Aging Male Rats. Chinese Medicine, 7, 37-44.

http://dx.doi.org/10.4236/cm.2016.72006 


\section{Late-Onset Hypogonadism, Rat}

\section{Introduction}

Late-onset hypogonadism ( $\mathrm{LOH}$ ), which is also described as partial androgen deficiency of the aging male (PADAM), is thought to be caused by testosterone deficiency in men with aging. It was reported that about $30 \%$ of males 50 years and older were suffering from LOH [1]. Many clinical symptoms are associated with $\mathrm{LOH}$, such as muscle weakness, obesity, osteoporosis, fatigue, insomnia, poor concentration, depression, and sexual dysfunction, indicating that $\mathrm{LOH}$ is a major public health threat for older men [2]. Testosterone replacement therapy (TRT) has become a popular option for LOH therapy. The sales volume of testosterone preparations keeps rising in the United States, and doubles between 2005 and 2010 [3]. Other parts of the world follow a similar trend [4]. The therapeutic effect of TRT is often satisfactory, but the risks of it should not be ignored. Some potential adverse effects of TRT were also reported, including erythrocytosis, cardiovascular events, gynecomastia, sleep apnea, prostate cancer, etc. [3]. It has been reported that intratesticular testosterone (ITT) is very important to normal spermatogenesis, and the ITT concentration is 50 - 100 times higher than serum testosterone levels [5]. Reducing ITT can lead to oligozoospermia and even azoospermia [6]. Supplying exogenous testosterone exerts a feedback inhibition on hypothalamic-pituitary-gonadal (HPG) axis, declines ITT concentration and suppresses spermatogenesis [7]. So, the $\mathrm{LOH}$ patients with future fertility desire should not adopt TRT. Finding a new method for testosterone supplementation would be very informative.

As a traditional herbal medicine, Guhanyangshengjing Tablet (GT) is widely used in China to nourish the kidney, strengthen the essence, delay senility, and relieve the symptoms of LOH such as palpitation, dizziness, fatigue, insomnia, and amnesia. But the effect of GT on testicular functions is not clear now. To provide empirical evidence for GT's clinical application in the treatment of LOH, we observed its therapeutic effects on testosterone synthesis and spermatogenesis, and investigated the potential mechanism by evaluating the expression of synaptonemal complex protein 3 (SYCP3), a meiotic marker, in the testis tissue of aging male rats.

\section{Materials and Methods}

\subsection{Drugs}

GT was purchased from Tus-Guhan group Hengyang Chinese medicine limited company. The main ingredients of GT are Herba Epimedii, Fructus Lycii, Fructus Ligustri Lucidi, Rhizoma Polygonati, Radix Paeoniae Alba, Semen Cuscutae, and Radix Ginseng. The tablets were powdered, suspended in distilled water and administrated via gavage.

\subsection{Animals and Experimental Design}

Forty adult male SD rats, weighing from 450 to 500 g, were provided by the Animal Center of Sun Yat-sen University (Guangzhou, China). The animals were 20 months of age and were housed individually under a 12 hour light/dark cycle at ambient temperature provided with water and food ad libitum. Rats were randomly allocated into four equal groups ( $\mathrm{n}=10$ per group) and administrated with GT daily at dose of $0,1.5 \mathrm{~g} / \mathrm{kg}, 3.0 \mathrm{~g} / \mathrm{kg}$, or $4.5 \mathrm{~g} / \mathrm{kg}$ respectively. Thirty days later, all of the rats were sacrificed and the reproductive organs were taken and weighted. Then, the analysis of serum testosterone, ITT, quality of sperm and immunohistochemical staining was performed.

The animal care strictly conformed to the guidelines of the Sun Yat-sen University Institutional Animal Care and Use Committee.

\subsection{Weighting of Body and Reproductive Organs}

Twenty-four hours later after the last gavage, rats were weighed, and anesthetized with ether. The blood samples were collected from the vena cava. Serum was separated for measurement of testosterone. Immediately after blood samples were collected, bilateral testes, epididymides, seminal vesicles and prostates were taken and weighed. 


\subsection{Testosterone Assays}

The serum was collected from each rat for quantitative determination of testosterone. About $50 \mathrm{mg}$ testicular tissues from the right were obtained from each rat and homogenized by sonication $(2 \mathrm{~s} \times 20 \mathrm{~s})$ in phosphate buffer solution (PBS). Then the tissues were centrifuged at 10,000 $\mathrm{g}$ for $10 \mathrm{~min}$ and the supernatants were collected for testosterone examination. Testosterone levels in samples from both sera and testicular tissues were measured using a commercially available enzyme-linked immunosorbent assay kit (R \& D Systems, Minneapolis, MN), following the manufacturer's instructions.

\subsection{Sperm Quality Analysis}

The excised epididymides from each rat were cut into small pieces, put in $1 \mathrm{ml} \mathrm{F12} \mathrm{media} \mathrm{containing} 0.1 \%$ bovine serum albumin and incubated in $34^{\circ} \mathrm{C}$ for at least 15 minutes to facilitate sperm transmigration from the epididymis. The numbers of sperm were counted twice using a hemacytometer and averaged. Fresh sperm was loaded onto a prewarmed glass slide to examine sperm motility by microscopy. The percentage of active sperm was counted to assay sperm motility.

\subsection{Immunohistochemical Analysis}

After the experiment, the left testes were dissected, fixed in Bouin's fixative for 12 hours and then embedded in paraffin. Testicular tissues were sectioned at $5 \mu \mathrm{m}$ thickness for immunohistochemical analysis.

In order to assay spermatogonial proliferation, the expression of a meiotic marker, SYCP3, was detected. Slides were incubated with a 1:500 dilution of the primary antibody (Rabbit anti-SCP-3 [Santa Cruz Biotechnology]). The SYCP3-positive cells in testicular tissue were detected by DAB (3,3'-diaminobenzidine tetrahydro chloride, Sigma) stain as depicted before [8]. The number of SYCP3-positive cells/tubule in cross-section of testes and percentages of SYCP3-positive tubules/section in the rat testes were both counted. At least 50 tubules per testis were counted. All counting procedure was conducted on an Olympus microscope (Center Valley, $\mathrm{PA})$.

\subsection{Statistical Methods}

Data were analyzed using one-way analysis of variance (ANOVA) which was carried out by SPSS 21.0 package (SPSS Inc., Chicago, IL, USA). Results were presented as mean \pm SEM. In all cases, probability values of $p$ less than 0.05 were considered statistically significant.

\section{Results}

\subsection{Changes in Body Weight and Reproductive Organs' Weights}

As shown in Figure 1, body weight did not change before and after the treatment of GT. The reproductive organs' weights showed an increasing tendency, but only the changes in $4.5 \mathrm{~g} / \mathrm{kg}$ group were statistically significant (testis: $1.55 \pm 0.17 \mathrm{~g}, p<0.05$; epididymides: $0.55 \pm 0.13 \mathrm{~g}, p<0.05$; seminal vesicles: $1.34 \pm 0.18 \mathrm{~g}, p<$ 0.05; and prostates: $0.52 \pm 0.08 \mathrm{~g}, p<0.01)$.

\subsection{Effects of GT on Concentrations of Serum Testosterone and ITT}

As shown in Figure 2, the serum testosterone levels of these rats increased after treatment with GT, and the result in $4.5 \mathrm{~g} / \mathrm{kg}$ group was significantly higher than the control $(5.96 \pm 1.33 \mathrm{ng} / \mathrm{ml} \mathrm{vs.} 4.89 \pm 0.87 \mathrm{ng} / \mathrm{ml}, p<$ 0.05). Similarly, the ITT concentrations in each group were also significantly improved after GT administration (the control group: $441.5 \pm 54.05 \mathrm{ng} / \mathrm{g}$; the $1.5 \mathrm{~g} / \mathrm{kg}$ group: $489.7 \pm 62.59 \mathrm{ng} / \mathrm{g}, p<0.05$; the $3.0 \mathrm{~g} / \mathrm{kg}$ group: $531.1 \pm 110.5 \mathrm{ng} / \mathrm{g}, p<0.05$; the $4.5 \mathrm{~g} / \mathrm{kg}$ group: $552 \pm 104.02 \mathrm{ng} / \mathrm{g}, p<0.05$ ).

\subsection{Semen Quality Was Improved after GT Treatment}

To determine whether the quality of semen changed after the treatment of GT, sperm number and motility were analyzed. As shown in Figure 3, the sperm number in groups treated with GT at dose of $3.0 \mathrm{~g} / \mathrm{kg}$ and $4.5 \mathrm{~g} / \mathrm{kg}$ 


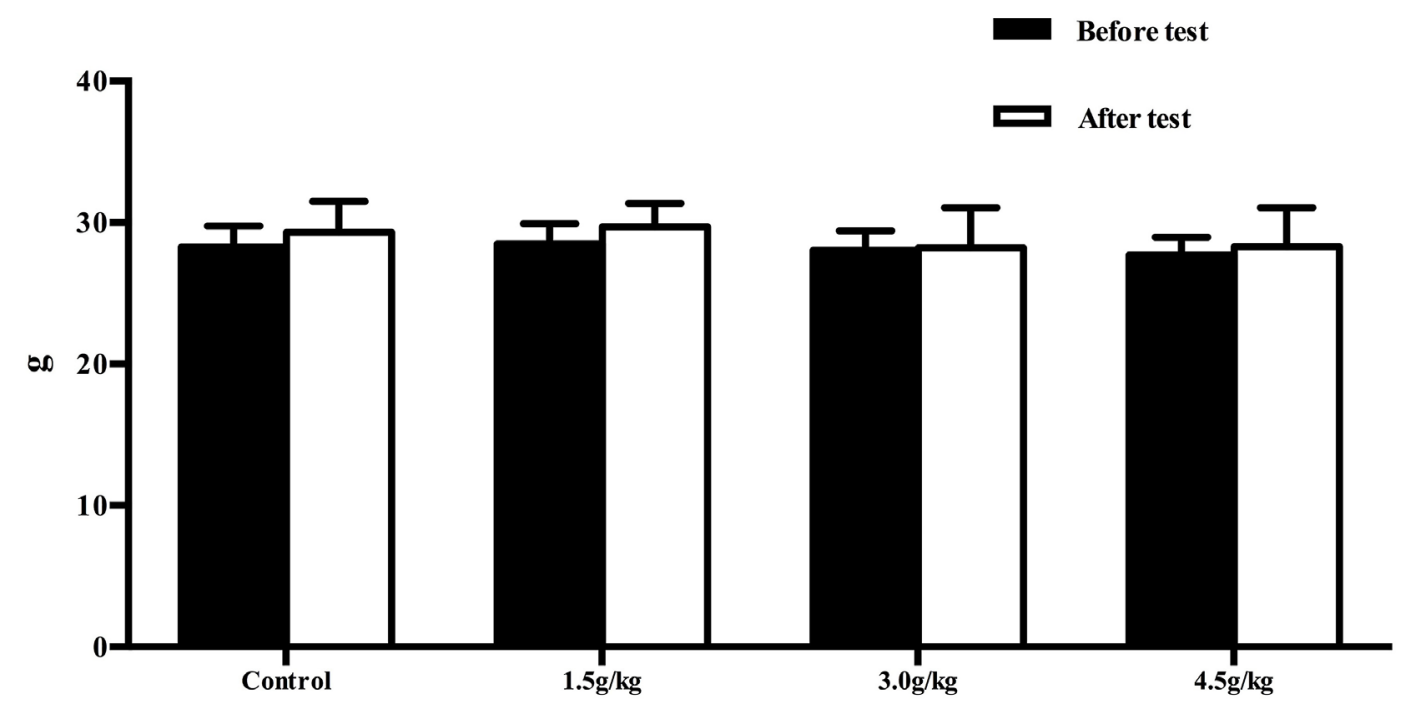

(a)

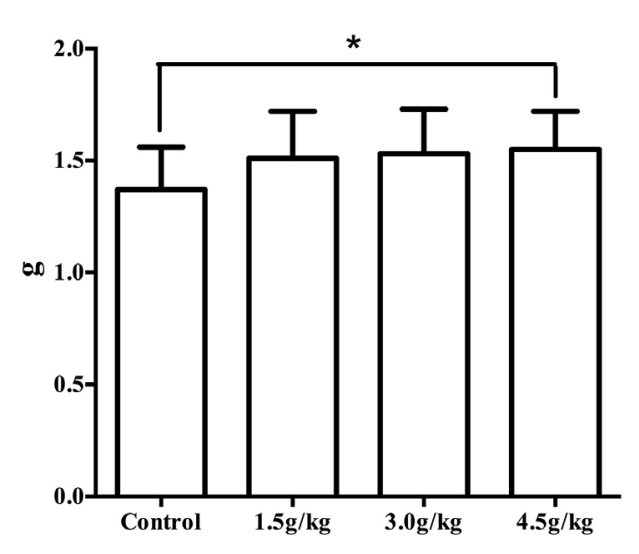

(b)

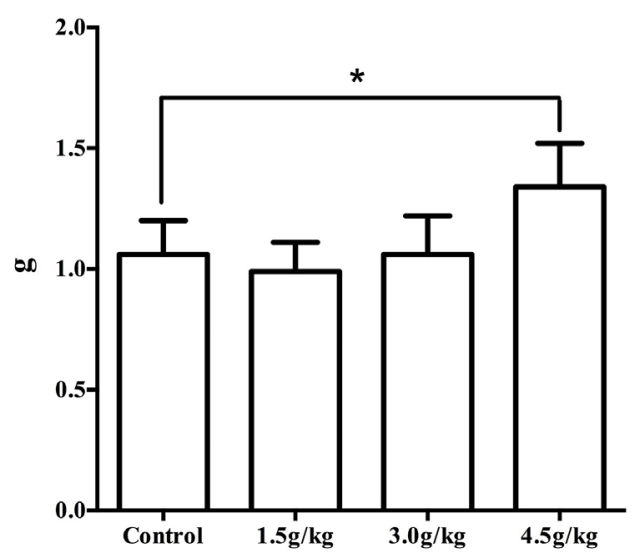

(d)

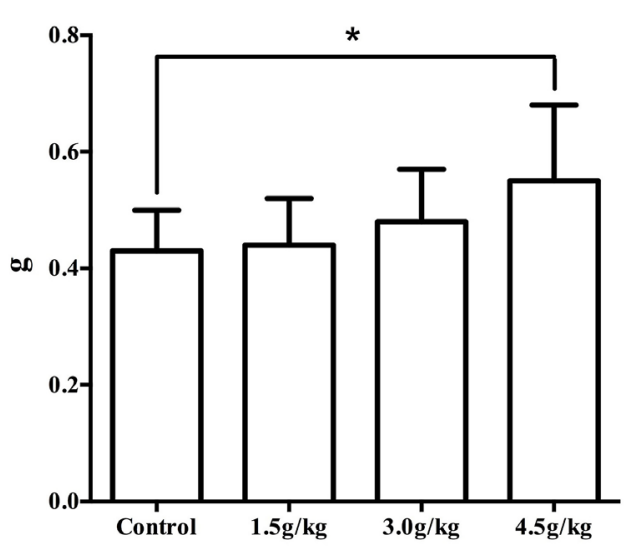

(c)

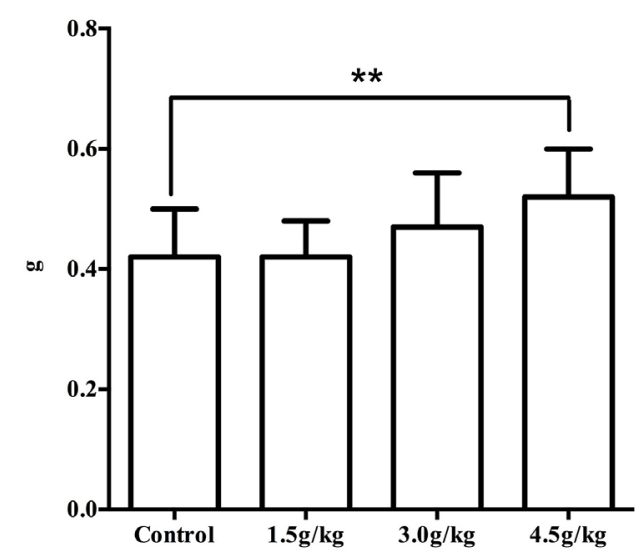

(e)

Figure 1. Effects of GT on body weight and reproductive organs' weights. (a) Body weight; (b) Testis weight; (c) Epididymidis weight; (d) Seminal vesicle weight; (e) Prostate weight. Data are shown as mean \pm SEM; $* p<0.05$, $* * p<0.01 ; \mathrm{n}=10 /$ group. 


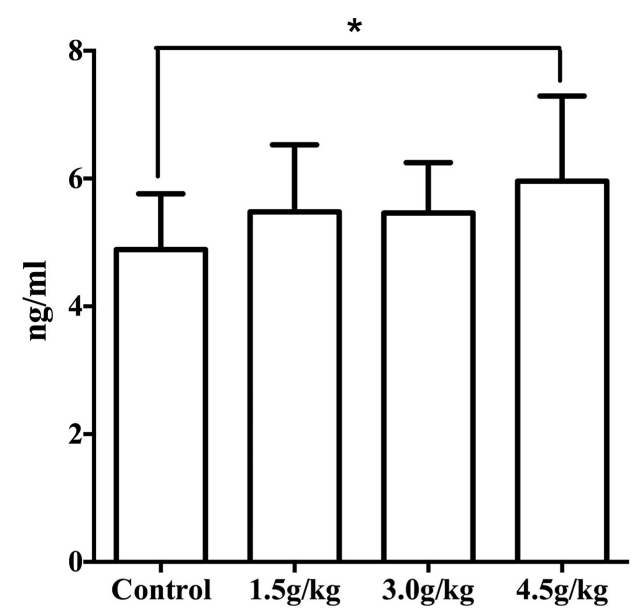

(a)

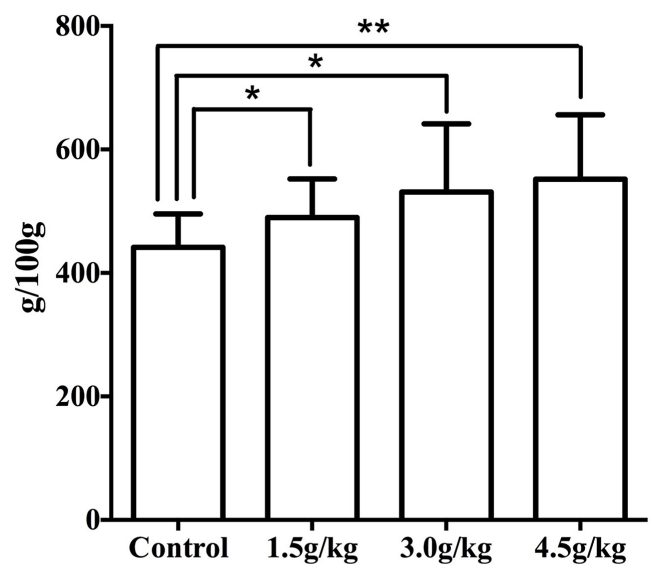

(b)

Figure 2. Quantification of testosterone concentrations after the administration of GT. (a) Serum testosterone levels; (b) ITT concentrations. Values represent the mean \pm SEM. ${ }^{*} p<0.05,{ }^{* *} p<0.01$; $=10$ /group.

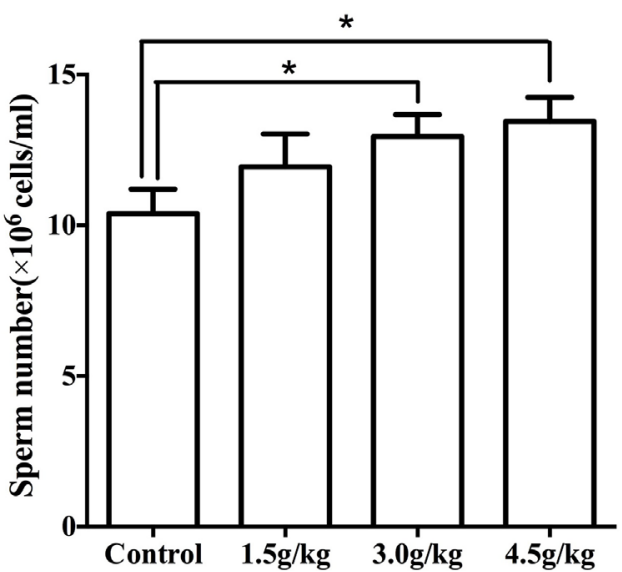

(a)

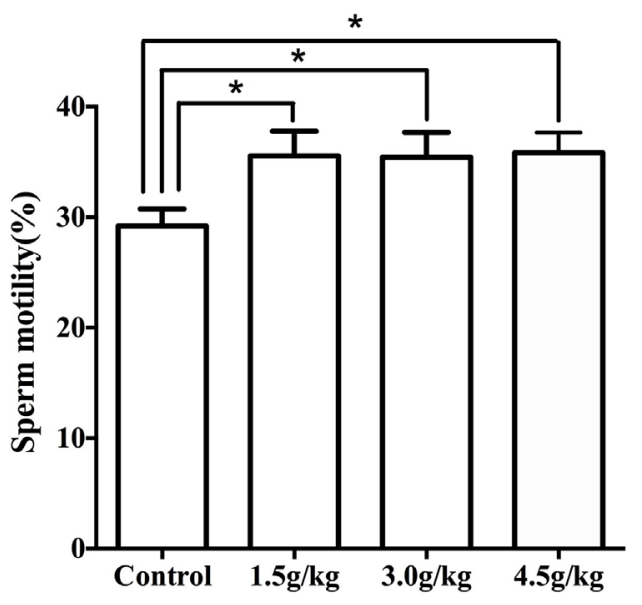

(b)

Figure 3. Alterations in semen quality after treated with GT. (a) Changes in sperm number; (b) Changes in sperm motility. Data are shown as mean $\pm \mathrm{SEM} ;{ }^{*} p<0.05 ; \mathrm{n}=10$ /group.

significantly increased $\left((13.74 \pm 2.78) \times 10^{6}\right.$ cells $/ \mathrm{ml}$, and $(14.34 \pm 2.65) \times 10^{6}$ cells/ml, respectively, both $p<$ 0.05). The improvements of sperm motility in all of the GT-treated groups were statistically significant ((35.56 \pm 7.02)\%, (35.43 \pm 7.12$) \%$, and (35.83 \pm 5.79$) \%$, respectively, all $p<0.05)$.

\subsection{Results of Immunofluorescence Staining}

The expression of SYCP3 was detected to assess the effect of GT on meiosis. As shown in Figures 4(a)-4(d), the distribution of SYCP3 increased in the GT-treated groups. According to the results of quantitative analysis, the number of SYCP3-positive cells/tubule cross-section increased in all of the GT-treated groups compared with the controls (the control group: $55.50 \% \pm 7.28 \%$; the $1.5 \mathrm{~g} / \mathrm{kg}$ group: $66.50 \% \pm 11.50 \%, p<0.05$; the 3.0 $\mathrm{g} / \mathrm{kg}$ group: $74.10 \% \pm 10.03 \%, p<0.01$; the $4.5 \mathrm{~g} / \mathrm{kg}$ group: $79.20 \% \pm 10.36 \%, p<0.01$ ) (Figure $4(\mathrm{e})$ ). The percentage of SYCP3-positive tubules/section were also increased after the GT administration, but only the change in the $4.5 \mathrm{~g} / \mathrm{kg}$ group was statistically significant (the control group: $75.96 \% \pm 6.92 \%$; the $1.5 \mathrm{~g} / \mathrm{kg}$ group: $80.01 \% \pm 6.35 \%, p>0.05$; the $3.0 \mathrm{~g} / \mathrm{kg}$ group: $78.98 \% \pm 9.78 \%, p>0.01$; the $4.5 \mathrm{~g} / \mathrm{kg}$ group: $85.04 \% \pm 6.69 \%$, $p<0.05$ (Figure 4(f)). 


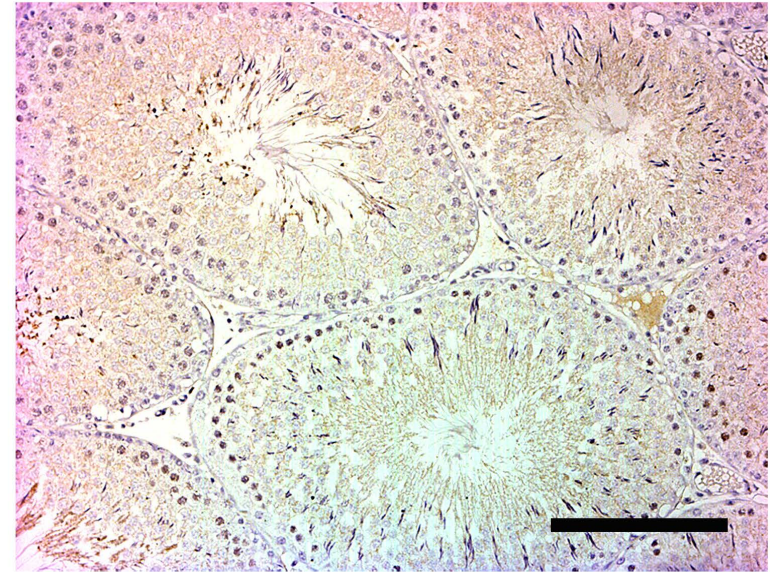

(a)

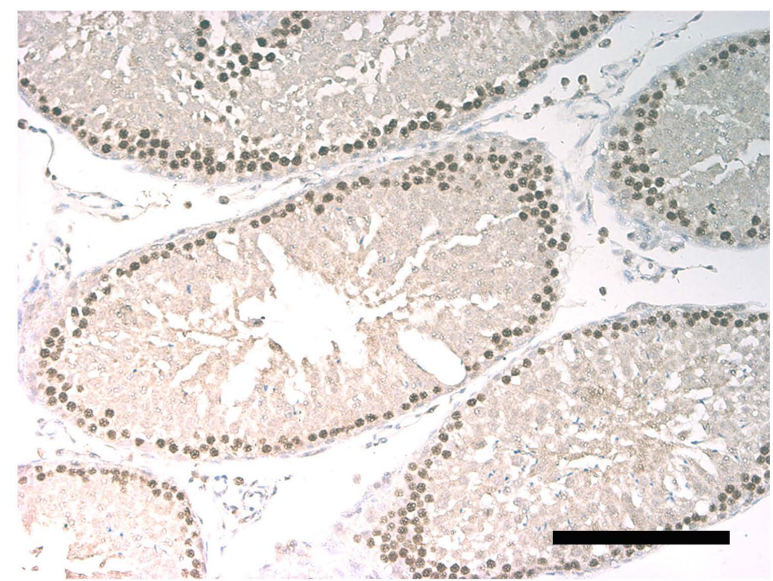

(c)

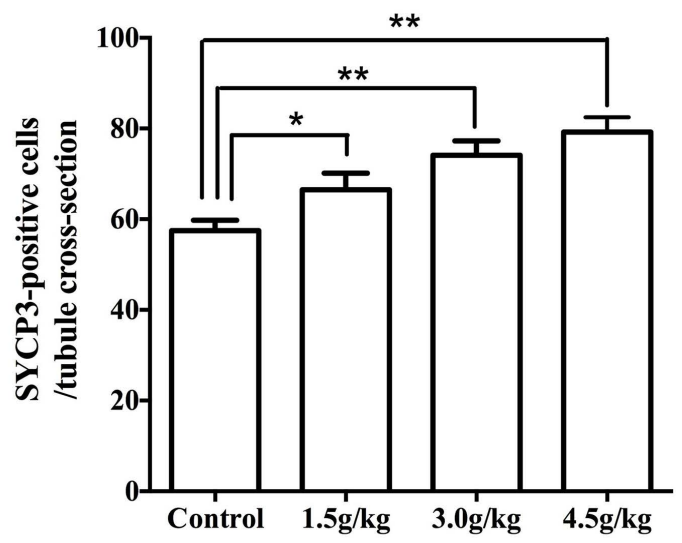

(e)

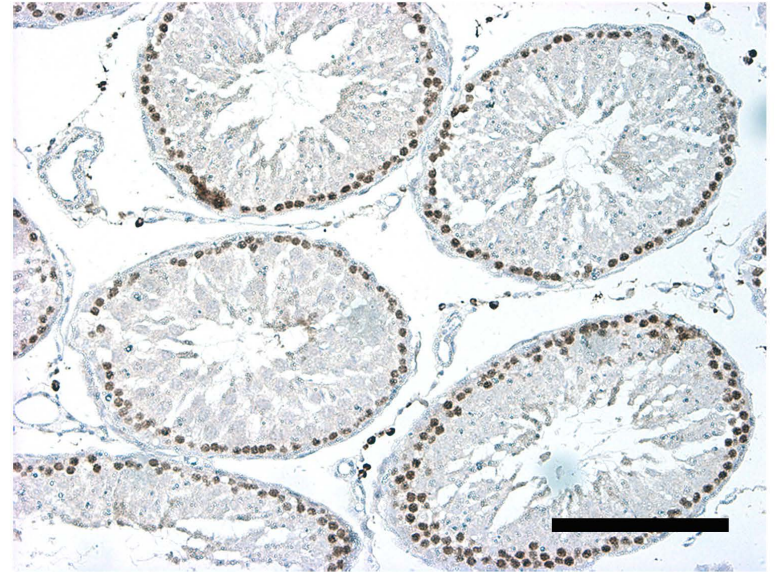

(b)

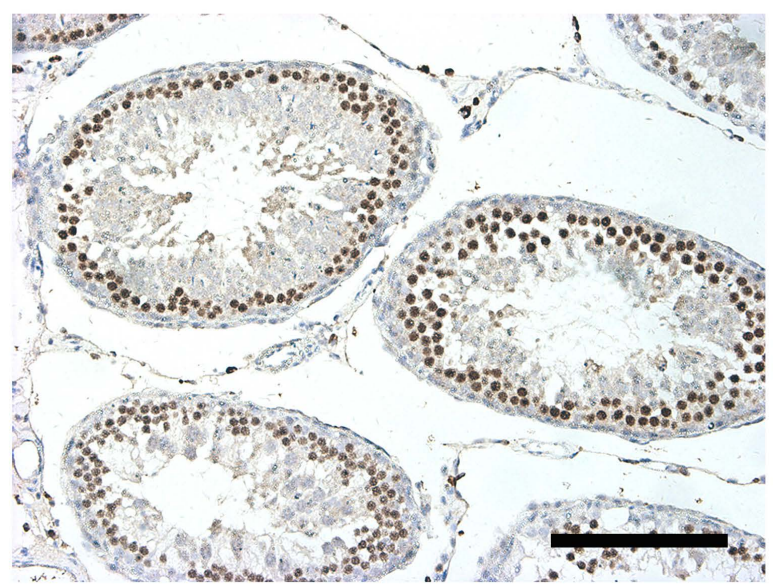

(d)

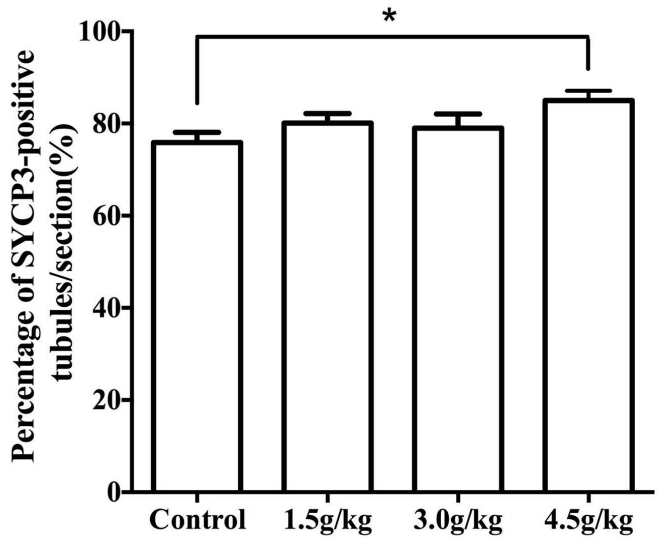

(f)

Figure 4. The SYCP3 expression in testis tissue of rats after GT administration. Immunohistochemical detection of germ cells immunopositive for SYCP3 is shown in (a) control group, (b) $1.5 \mathrm{~g} / \mathrm{kg}$ group, (c) $3.0 \mathrm{~g} / \mathrm{kg}$ group, and (d) $4.5 \mathrm{~g} / \mathrm{kg}$ group. (e) Quantification of immunopositive cells per tubule cross-section is shown. (f) Comparison of percentage of SYCP3-positive tubules/section between different groups. Data are shown as mean $\pm \mathrm{SEM} ;{ }^{*} p<0.05,{ }^{* *} p<0.01 ; \mathrm{n}=10$ /group; bars = 50 $\mu \mathrm{m}$. 


\section{Discussion}

In the present study, we demonstrated that the administration of GT could improve the weights of reproductive organs, increase the testosterone synthesis in vivo and enhance the expression of SYCP3 in testes of aging male rats.

It has been reported that serum testosterone concentrations gradually decline with age in males, which lead to the occurrence of $\mathrm{LOH}$ [9] [10]. LOH is a clinical and biochemical state with advancing age, characterized by typical symptoms and low serum testosterone levels [11]. One study have proposed the minimum criteria for the diagnosis of LOH based on the simultaneous occurrence of three sexual symptoms (decreased sexual desires and morning erections, and erectile dysfunction) and total testosterone below $11 \mathrm{nmol} / \mathrm{L}$ and free testosterone below $220 \mathrm{pmol} / \mathrm{L}$ [12]. Age-related testosterone deficiency is thought to be associated with weight gain and deterioration of general health owing to some chronic diseases, such as diabetes, cardiac, hepatic or renal failure, hypertension, and inflammatory arthritis etc. [3]. Pye et al. has declared that severe LOH was associated with substantially higher risks of cardiovascular mortality, to which both the testosterone concentration and the presence of sexual symptoms contributed independently [10]. Testosterone replacement is widely used for the treatment of $\mathrm{LOH}$, but, as mentioned above, its short- and long-term risks really exist and should not be ignored.

Traditional Chinese Medicine plays an important role in the treatment of LOH in China, Japan and Korea. GT is manufactured according to Yangshengfang, an ancient Chinese medical book about 2000 years ago. As depicted above, GT is composed of 12 ingredients and can tonify qi, essence and marrow, nourish the blood, and invigorate bones and tendons. It has been reported that GT can relieve symptoms relative to LOH including osteoporosis, fatigue, and atherosclerosis [13]-[15]. Zhang YW et al. demonstrated that GT can favorably change the level of hormone and some biochemical indexs in the 20-month-old female rats [16]. Wen ZJ et al. proved that GT can decrease the percentage of distortion and lipid peroxidation in body [17]. But, to the best of our knowledge, the effects of GT on spermatogenesis and testosterone synthesis have not yet been elucidated. In the present study, we selected twenty-month-old male rats as an LOH animal model for examining the effects of GT on spermatogenesis and testosterone levels in vivo.

After the experiment, the weight of body and reproductive organs were measured. No significant change in body weight was observed after GT treatment. We found that testis weight increased after the treatment of GT, which indicated that the function of testis might have been strengthened. This was further proved by the improvement of ITT and serum testosterone level after GT administration. Simultaneously, the weights of epididymides, seminal vesicles and prostates also increased, but only the changes in $4.5 \mathrm{~g} / \mathrm{kg}$ group was statistically significant, which indicated that GT might work in a dose-dependent manner. This phenomenon is presumed to be caused by the increase of serum testosterone concentration.

We continued to examine the changes of semen quality in epididymis and found that the sperm number and motility increased significantly after the administration of GT, which is thought to be due to the enhanced ITT concentrations caused by GT. In order to determine whether GT treatment affect spermatogenesis, we thereby observed the expression of a meiotic marker, SYCP3, by immunohistochemical analysis. As a result, we detected that the SYCP3 expression was significantly enhanced in the GT-treated groups, especially in the $4.5 \mathrm{~g} / \mathrm{kg}$ group, when compared with the control group. The increase in the number of germ cells immunopositive for a differentiation marker, SYCP3 after GT exposure suggests that GT treatment induced germ cell differentiation in vivo. Our results also indicate that GT can improve meiotic spermatogenesis in a dose-dependent manner. During the experiment, the weights of rats didn't change obviously, and no serious adverse symptoms associated with GT administration were found, which indicated that treatment with GT might be quite safe for LOH patients.

\section{Conclusion}

In conclusion, our results indicated that GT might have a positive role in function of Leydig cells. The administration of GT can enhance testosterone concentrations in vivo, relieve LOH symptoms, and improve spermatogenesis. GT might be a safe therapy for LOH patients with a future fertility desire.

\section{References}

[1] Allan, C.A. and McLachlan, R.I. (2004) Age-Related Changes in Testosterone and the Role of Replacement Therapy in 
Older Men. Clinical Endocrinology (Oxford), 60, 653-670. http://dx.doi.org/10.1111/j.1365-2265.2004.02002.X

[2] Zang, Z.J., Ji, S.Y., Dong, W., Zhang, Y.N., Zhang, E.H. and Bin, Z. (2015) A Herbal Medicine, Saikokaryukotsuboreito, Improves Serum Testosterone Levels and Affects Sexual Behavior in Old Male Mice. Aging Male, 18, 106-111. http://dx.doi.org/10.3109/13685538.2014.963042

[3] Huhtaniemi, I. (2014) Late-Onset Hypogonadism Current Concepts and Controversies of Pathogenesis, Diagnosis and Treatment. Asian Journal of Andrology, 16, 192-202. http://dx.doi.org/10.4103/1008-682X.122336

[4] Handelsman, D.J. (2012) Pharmacoepidemiology of Testosterone Prescribing in Australia, 1992-2010. Medical Journal of Australia, 196, 642-645. http://dx.doi.org/10.5694/mja11.11277

[5] Kim, E.D., Crosnoe, L., Bar-Chama, N., Khera, M. and Lipshultz, L.I. (2013) The Treatment of Hypogonadism in Men of Reproductive Age. Fertility and Sterility, 99, 718-724. http://dx.doi.org/10.1016/j.fertnstert.2012.10.052

[6] McLachlan, R.I., O’Donnell, L., Meachem, S.J., Stanton, P.G., de K., Pratis, K. and Robertson, D.M. (2002) Hormonal Regulation of Spermatogenesis in Primates and Man: Insights for Development of the Male Hormonal Contraceptive. Journal of Andrology, 23, 149-162.

[7] Moss, J.L., Crosnoe, L.E. and Kim, E.D. (2013) Effect of Rejuvenation Hormones on Spermatogenesis. Fertility and Sterility, 99, 1814-1820. http://dx.doi.org/10.1016/j.fertnstert.2013.04.003

[8] Varghese, F., Bukhari, A.B., Malhotra, R. and De, A. (2014) IHC Profiler: An Open Source Plugin for the Quantitative Evaluation and Automated Scoring of Immunohistochemistry Images of Human Tissue Samples. PLoS ONE, 9, e96801. http://dx.doi.org/10.1371/journal.pone.0096801

[9] Feldman, H.A., Longcope, C., Derby, C.A., Johannes, C.B., Araujo, A.B., Coviello, A.D., Bremner, W.J. and McKinlay, J.B. (2002) Age Trends in the Level of Serum Testosterone and Other Hormones in Middle-Aged Men: Longitudinal Results from the Massachusetts Male Aging Study. Journal of Clinical Endocrinology \& Metabolism, 87, 589598. http://dx.doi.org/10.1210/jcem.87.2.8201

[10] Pye, S.R., Huhtaniemi, I.T., Finn, J.D., Lee, D.M., O’Neill, T.W., Tajar, A., Bartfai, G., Boonen, S., Casanueva, F.F., Forti, G., Giwercman, A., Han, T.S., Kula, K., Lean, M.E., Pendleton, N., Punab, M., Rutter, M.K., Vanderschueren, D. and Wu, F.C. (2014) Late-Onset Hypogonadism and Mortality in Aging Men. Journal of Clinical Endocrinology \& Metabolism, 99, 1357-1366. http://dx.doi.org/10.1210/jc.2013-2052

[11] Bhasin, S., Cunningham, G.R., Hayes, F.J., Matsumoto, A.M., Snyder, P.J., Swerdloff, R.S. and Montori, V.M. (2010) Testosterone Therapy in Men with Androgen Deficiency Syndromes: An Endocrine Society Clinical Practice Guideline. The Journal of Clinical Endocrinology and Metabolism, 95, 2536-2559. http://dx.doi.org/10.1210/jc.2009-2354

[12] Wu, F.C., Tajar, A., Beynon, J.M., Pye, S.R., Silman, A.J., Finn, J.D., O’Neill, T.W., Bartfai, G., Casanueva, F.F., Forti, G., Giwercman, A., Han, T.S., Kula, K., Lean, M.E., Pendleton, N., Punab, M., Boonen, S., Vanderschueren, D., Labrie, F. and Huhtaniemi, I.T. (2010) Identification of Late-Onset Hypogonadism in Middle-Aged and Elderly Men. The New England Journal of Medicine, 363, 123-135. http://dx.doi.org/10.1056/NEJMoa0911101

[13] Liu, L.Y., Sun, Z.L., Tang, X.J. and Shou, D.W. (1995) Experimental Research in Anti-Atherosclerosis Effect of Guhanyangshengjing. Hunan Journal of Traditional Chinese Medicine, 11, 35-37.

[14] Ge, C.S., Wang, S.F. and Yong, L. (1997) Effect of Guhanyangshengjing on Osteoporosis: Clinical Analysis of 35 Cases. Hunan Guiding Journal of Traditional Chinese Medicine \& Pharmacology, 3, 9-10.

[15] Chen, X.D., Wang, B.H. and Wang, B.B. (1999) Study on Antifatigue Effect and Potential Mechanisms of Ancient Han Health-Keeping. Hunan Guiding Journal of Traditional Chinese Medicine \& Pharmacology, 5, 29-30.

[16] Zhang, Y.W., Tu, J.W. and Chen, Y.X. (2004) Effects of Guhanyangshengjing Solution on Serum NO, Sex Hormone and SOD in the 20-Month-Old Female Rats. Journal of Beijing University of Traditional Chinese Medicine, 27, 49-51.

[17] Wen, Z.J. and Chen, X.C. (2007) Effect of Ancient Han Health Essence of Red Cell Morphology and Free Radical Metabolism in Exercise Mice. Journal of Zhangjiang Normal College, 28, 104-107. 